\title{
Chaperone-Mediated Autophagy Plays an Important Role in Regulating Retinal Progenitor Cell Homeostasis
}

Caixia Jin

Tongji University

Qingjian Ou

Tongji University https://orcid.org/0000-0002-6881-8680

Jie Chen

Tongji University

Tao Wang

Tongji University

Jieping Zhang

Tongji University

Zhe Wang

Tongji University

Yuanyuan Wang

Tongji University

Haibin Tian

Tongji University

Jing-Ying Xu

Tongji University

Furong Gao

Tongji University

Juan Wang

Tongji University

Jiao Li

Tongji University

Lixia Lu

Tongji University

Guo-Tong Xu ( $\nabla$ gtxu@tongji.edu.cn )

Tongji University 
Keywords: Retinal Progenitor Cells, Autophagy, Lysosome, IFITM3, Cell Proliferation

Posted Date: November 30th, 2021

DOI: https://doi.org/10.21203/rs.3.rs-1076949/v1

License: (c) (1) This work is licensed under a Creative Commons Attribution 4.0 International License. Read Full License

Version of Record: A version of this preprint was published at Stem Cell Research \&amp; Therapy on April 1st, 2022. See the published version at https://doi.org/10.1186/s13287-022-02809-z. 


\section{Abstract}

Purpose: Autophagy is a key regulator of stem cell quiescence and self-renewal, especially in mesenchymal stem cells but related research on neural retinal stem cells is still limited. We are aimed to explore the function and mechanism of autophagy in the neural retinal stem cell.

Methods: The published single cell sequencing data was involved to analysis the expression time course of IFITM3 in the mouse neural retinal progenitor cells (mNRPCs). The RNA interference was used to knock down the expression of IFITM3 in the mNRPCs. And the normal mNRPCs and mNRPCs with knockdown of IFITM3 were analysis with the CCK8 for the cell viability, RNA-seq for the mRNA expression, real-time quantitative PCR, immunofluorescence assay for the location of relative proteins, western blot for the levels of relative proteins and autophagy flux assay.

Results: This study showed the mNRPCs in vivo and in vitro high expressed IFITM3 which are expressed in the mNRPCs. The proliferation of mNRPCs was greatly inhibited, and cell viability was greatly reduced after IFITM3 knockdown. Moreover, RNA-seq analysis showed that lysosomes were significant changed after IFITM3 knockdown. When cells were treated with rapamycin (RAMP), lysosome activation and agglomeration were evident in all groups. However, there was no significant difference between IFITM3 knockdown groups. The expression of LAMP1 was significantly increased, accompanied by increased lysosome agglomeration, in RAMP-treated cells and especially in IFITM3-knockdown cells. Further detection showed that SQSTM1/p62, HSC70 and LAMP-2A were upregulated, while there was no significant difference in LC3A/B expression, which demonstrated that the MA pathway was not activated but the CMA pathway was activated when knockdown of IFITM3.

Conclusion: Our findings indicate that IFITM3 participates in regulating MNRPC viability and proliferation mainly through the CMA pathway, indicating that IFITM3 plays a significant role in maintaining the homeostasis of progenitor cell self-renewal by sustaining low-level activation of the CMA pathway to eliminate factors that are deleterious to cells and acts as a very important protector of RPCs.

\section{Introduction}

Retinal progenitor cells (RPCs) have been investigated for years, and their transplantation or endogenous activation is a promising potential therapeutic avenue in the future [1, 2]. In our previous study, we found that the mouse neural retinal progenitor cells (mNRPCs) can be maintained and efficiently amplified in vitro for long periods [3].

Autophagy is one of many mechanisms involved in regulating the homeostasis of eukaryotic cells [48]and plays an important role in maintaining cell proliferation and self-renewal, especially in mesenchymal stem cells (MSCs) [9-11]. RNA-seq analysis of mNRPCs indicated that interferon-induced transmembrane protein 3 (IFITM3) plays an important role in mNRPC proliferation in vitro. IFITM3 is a transmembrane protein that localizes to several cellular components, including the apical part of the cell, cell surface and endoplasmic reticulum (ER), and is well known for its role in modulating the interferon- 
mediated innate immune system to defend against invading pathogenic viruses [12-17]. In addition to its role in regulating immune cells $[18,19]$, IFITM3 is involved in germ cell homing and maturation during embryonic development [20-22]. However, the mechanism by which it regulates progenitor cell proliferation has not yet been reported.

Previously, we reported that the non-canonical Wnt5A/Ca2+ pathway may participate in regulating RPC self-renewal during in vitro culture. Here, we investigated whether the knockdown of IFITM3 expression would markedly inhibit RPC proliferation and cell viability through the chaperone-mediated autophagy (CMA) pathway. Our study suggested that IFITM3 is a gatekeeper of progenitor cells that keeps the cells healthy during growth and maintain their self-renewal. When IFITM3 was knocked down in mNRPCs, the cells showed a significant decrease in proliferation, as IFITM3 knockdown disrupted cell membrane transport systems and destabilized cell membranes, and the CMA pathway was activated in this process. RNA-seq data suggest that other regulatory factors, such as amino acid metabolism and fat metabolism, were also involved in regulating proliferation after IFITM3 knockdown, which suggests that inhibition of the expression of IFITM3 caused destruction of the cell membrane and eventually induced a metabolic crisis resulting in cell death. Taken together, these data indicate that IFITM3 is the first line of protection for RPC homeostasis and regulates MNRPC viability and proliferation mainly through the CMA pathway.

\section{Materials And Methods}

\section{Cell Culture and RNA Interference}

Adult mouse neural RPCs (mNRPCs) established by our laboratory as our previous description[3] were plated onto dishes coated with 2\% Matrigel (Corning, USA) and cultured in 1× N2/B27 (Thermo Fisher Scientific, USA), $10 \mathrm{ng} / \mathrm{ml}$ bFGF (PeproTech, USA), $2 \mu \mathrm{m}$ CHIR99021 (Selleck Chemicals, USA) and 0.11 mM 2-Mercaptoethanol (Thermo Fisher Scientific, USA) in DMEM/F12 medium (all other materials were from Thermo Fisher Scientific-Gibco, USA). To knock down IFITM3, commercially validated IFITM3 siRNA was synthesized by Proteintech (Rosemont, IL, USA) and transfected into cells using Lipofectamine 3000 (Thermo Fisher Scientific, USA) according to the manufacturer's protocol. After transfection for approximately $48 \mathrm{~h}$, the cells were collected for further assays. The targeted sequences for the experiments are listed in Supplementary Table 1.

\section{Cell Viability Assay}

For the cell viability assay, cells were seeded at a density of $1 \times 10^{5}$ cells per $100 \mu$ in each well in 96 -well microtiter plates (Corning, USA) and transfected with siRNA for $48 \mathrm{~h}$. Cells were grown in each medium in triplicate. Then, $10 \mu \mathrm{l}$ of reagent from the Cell Counting Kit-8 (CCK-8, TargetMol, USA) was added to each well, and the plates were incubated for $3 \mathrm{~h}$ at $37^{\circ} \mathrm{C}$. Cell viability was measured as the absorbance at 450 $\mathrm{nm}$ with a microplate reader (iMark ${ }^{\mathrm{TM}}$ Microplate Absorbance Reader). The mean optical density (OD) values from triplicate wells containing each culture medium were used as the cell viability indices.

\section{Real-time Quantitative PCR}


To obtain total RNA, cells were lysed with TRIzol reagent and treated with RNase-free DNase I (both from TaKaRa, China). Reverse transcription (RT) was performed according to our previously published article[3]. Then, quantitative RT-RCR was performed using the SYBR Green Master Mix system (Tiangen Biotech, China). Each PCR mixture contained $10 \mu \mathrm{l}$ of $2 \times$ SYBR Green Master Mix, sense and antisense primers (Sangon Biotech, China) at $5 \mu \mathrm{mol} / \mu \mathrm{l}, 10 \mathrm{ng}$ of cDNA in a total volume of $20 \mu \mathrm{l}$ and was subjected to the following protocol: 45 cycles of $95^{\circ} \mathrm{C}$ for $15 \mathrm{~s}, 60^{\circ} \mathrm{C}$ for $30 \mathrm{~s}$ and $72^{\circ} \mathrm{C}$ for $30 \mathrm{~s}$. For relative quantification, $2^{-\triangle \Delta C t}$ values were calculated and used as an indication of relative expression levels. The IFITM3 gene primer sequences used for RT-PCR were 5'-TGTCCAAACCTTCTTCTCTCC-3' and 5 '-CGTCGCCAACCATCTTCC-3'. The internal control primer sequences for $\beta$-actin were $5^{\prime}$ -

GTGGACATCCGCAAAGAC-3' and 5'- AAAGGGTGTAACGCAACTA-3'.

\section{Immunofluorescence Assay}

Cells were fixed in $4 \%$ paraformaldehyde (PFA, Sigma-Aldrich, Germany) following the procedure described in our previously published article[3]. At the end of the experiment, the cells were stained with compatible Alexa 488- or Alexa 555-conjugated secondary antibodies in PBS for $30 \mathrm{~min}$ at room temperature. The antibodies are listed in Supplementary Table 2.

\section{Western Blot Analysis}

Cells were harvested and homogenized in RIPA buffer (Beyotime, China) supplemented with protease and phosphatase inhibitor cocktails (TargetMol, USA) on ice and subjected to a previously described protocol[3]. The antibodies used are listed in Supplementary Table 2.

\section{Bioinformatics Analysis}

For the single cell sequence analysis, all cells in the website (https://github.com/gofflab/developing_mouse_retina_scRNASeq) were clustered and visualized using the method of uniform manifold approximation and projection (UMAP) with Seurat (v4.0.4)::RunUMAP $[23,24]$. The annotations of all cells were provided in the dataset. The expression of IFITM3 gene in each sample were visualized using the function FeaturePlot in R package Seurat (v4.0.4).

For the RNA sequence analysis, RNA was extracted from mNRPCs and IFITM3-knockdown cells at $48 \mathrm{~h}$ with TRIzol reagent (TaKaRa, China). The RNA-seq library was sequenced with an Illumina NovaSeq 6000 $\mathrm{PE} 150$. The standard parameters $|\log \mathrm{FC}|>1$ and $\mathrm{P}<0.05$ were used to screen differentially expressed genes (DEGs). The R package clusterProfiler was used for Gene Ontology (GO) and Kyoto Encyclopedia of Genes and Genomes (KEGG) analyses. The results were visualized with GOplot.

\section{Organelles Involved in IFITM3-Knockdown Cells}

After $48 \mathrm{~h}$ of transfection with IFITM3 siRNA, cells were treated with or without $100 \mathrm{nM}$ rapamycin (RAMP, Selleck, USA) for $4 \mathrm{~h}$ then analyzed after incubation with $50 \mathrm{nM}$ LysoTracker (Beyotime, China), $50 \mathrm{nM}$ MitoTracker (Beyotime) and $1 \mu \mathrm{M}$ ER-Tracker (Beyotime) for $30 \mathrm{~min}$. The nuclei were stained with Hoechst 33342 (Sigma-Aldrich, Germany). 


\section{LC3-GFP-mCherry Construction and Infection and Autophagy Flux Assay}

The Ad-mCherry-GFP-LC3 plasmid was purchased from Addgene (plasmid \#110060), amplified and sequenced following instructions from Addgene using the following sequencing primers: forward: $5^{\prime}$ CGCGGATCCGGTCGCCACCATGGTGAGCAAGGGCGAG-3', reverse: 5'-

CGCGGCGCGCCGCTGGGTCTAGATGCATGC-3'. The PCR fragment was sequenced to verify that no errors had been introduced. LC3-GFP-mCherry lentivirus was produced by transfection in 293T cells.

The virus-containing supernatant was collected after $48 \mathrm{~h}$ of transfection and subjected to a previously described protocol[3]. GFP and mCherry expression was verified by microscopy. The cells were treated with $100 \mathrm{nM}$ RAMP to study autophagic flux after IFITM3 knockdown for $48 \mathrm{~h}$. Then, the expression of GFP and mCherry was observed after staining with Hoechst 33342 (Sigma-Aldrich, Germany).

\section{Statistical Analysis}

All data are expressed as the mean \pm SEM. All analyses were performed with GraphPad Prism 6 software. One-way ANOVA was employed for the statistical comparisons. A value of $\mathrm{P}<0.05$ was considered to indicate statistical significance.

\section{Results}

\section{IFITM3 is involved in mNRPC proliferation}

Initially, to obtain the variation and distribution of IFITM3 gene during the development of mouse retina in vivo, we integrated a published dataset of the developing murine retina in single cell RNA-sequencing and visualized the cell-type identification in Figure 1A[24]. During the embryonic stage, the IFITM3 gene was mainly expressed in the early RPCs at embryological day 11(E11) and E12 (Figure 1B). For the postnatal stage (P0, P2, P8), fewer positive expressed in the detected cells, while for the postnatal day 14 , the RPCs and muller cells also are positive expression of IFITM3. These results suggest the IFITM3 are important for the retinal progenitor cells in the retina development.

To investigate the function of IFITM3 in mNRPCs, we transfected siRNA IFITM3 into mNRPCs and examined cell viability. Cell growth (Figure 1C-1E and Supplementary Figure S1A-1C) was clearly inhibited in the IFITM3 siRNA transfected group (siRNA IFITM3) compared with c group and scramble control (SC) group, with significantly decreased expression of IFITM3 at the RNA (Figure 1F) and protein levels (Figure 1G) after IFITM3 knockdown, but there was no significant decrease in ARPE19 cell growth (Supplementary Figure S1D-E). The cell viability was greatly decreased after IFITM3 knockdown, shown by the CCK-8 assay, significantly differed among the groups (Figure $1 \mathrm{H}$ ), and the protein expression of Cyclin D1 (CCND1) decreased in the corresponding cell groups (Figure 1I), These results indicated that IFITM3 is involved in RPC proliferation through the regulation of cell division and cell viability in vivo and in vitro. 


\section{Cell membrane structure and function were deeply changed after IFITM3 knockdown}

To further explore the regulatory effect of IFITM3 knockdown on the cells, RNA-seq analysis was performed. Among the $\mathrm{GO}$ terms, cell membrane and extracellular matrix (ECM)-related, transmembrane transport-related and synaptic transmission enrichment-related terms were the most enriched after knockdown of the IFITM3 gene. These results revealed that genes related to ion transmembrane transport, vacuoles, lysosomes and cellular calcium ion homeostasis were upregulated in the knockdown group, while genes involved in regulation of the ECM, WNT pathway and cell proliferation were significantly downregulated (Figure $2 \mathrm{~A}$ ), suggesting that the stability of the cell membrane system was seriously damaged and that its material transport function was blocked, ultimately leading to cell death due to a lack of nutrients, and these findings further reveal how IFITM3 is involved in regulating mNRPC proliferation. The KEGG pathway enrichment assay further revealed that the signaling pathways enriched in the DEGs mainly included the synaptic vesicle cycle, the lysosome pathway, the calcium signaling pathway, the MAPK signaling pathway and other upregulated pathways (Figure 2B and Supplementary Figure S2A), as well as downregulated pathways, such as the WNT, amino acid biosynthesis and fatty acid metabolism pathways, among others (Figure 2C and Supplementary Figure S2B).

Furthermore, $\mathrm{GO}$ analysis of the ECM pathway showed that the expression of ion channel-related genes, including the genes encoding calcium (such as CACNB2), potassium (such as KCNB1, KCNF1 and KCNC3) and chloride (such as CLCN1) ion channels, was clearly changed after IFITM3 knockdown (Supplementary Figure S2C); additionally, genes that regulate cell proliferation involved in metabolism (such as TNC and MVD) and proliferation-related pathway genes (such as CCND3, STAT1 and IL6) (Supplementary Figure S2D) also showed significant changes in expression, indicating that inhibition of IFITM3 expression led to damage to the cell membrane, abnormal material transport, abnormal metabolism of intracellular substances, decreased cell proliferation and ultimately cell death. Taken together, these results indicate that the integrity of the membrane system (including the cell membrane, vacuole, lysosome and ECM) was impaired after IFITM3 knockdown and that cells could no longer survive without sufficient nutrition, eventually leading to cell death.

\section{Lysosome Activation in IFITM3-Knockdown Cells}

Because membrane-related systems were severely damaged after IFITM3 knockdown, we studied membranous organelles, including the endoplasmic reticulum (ER), mitochondria and lysosomes, in the cell. The lysosome is an important site of regulation for mTORC1 signaling, which mainly controls eukaryotic cell growth. We treated cells with $100 \mathrm{nM}$ RAMP, a specific mTOR inhibitor and an autophagy activator, and then treated the cells with $50 \mathrm{nM}$ LysoTracker, $50 \mathrm{nM}$ MitoTracker and $1 \mu \mathrm{M}$ ER-Tracker for 30 min. Lysosomes showed agglomeration in the IFITM3-knockdown cells without RAMP treatment, while lysosomes showed agglomeration in all groups when with RAMP treatment, but there was no significant difference in IFITM3-knockdown cells with or without RAMP treatment (Figure 3A). Mitochondria and the ER were increased in IFITM3-knockdown cells treated with RAMP, but there was no significant difference 
among the groups (Figure 3B-C), suggesting that mitochondria and the ER were not involved in regulating mNRPC proliferation after IFITM3 knockdown and microautophagy $(\mathrm{mA})$ was not activated.

The lysosome-specific markers lysosome-associated membrane protein 1 (LAMP1) and LAMP2A were detected in RAMP-treated and untreated cells, which showed that the expression of LAMP1 and LAMP2A was significantly increased and accompanied by increased lysosome agglomeration in RAMP-treated cells, especially IFITM3-knockdown cells (Figure 4A-C), indicating activation of the lysosome system followed by destruction of the cell membrane system to initiate cell cleanup, eventually causing cell death. These results suggest that IFITM3 in the cell membrane provides the first protective barrier for mNRPCs, and its decreased expression leads to the breakdown of cell homeostasis and initiation of lysosome formation to eliminate damaged cells. Furthermore, there was no significant difference among the IFITM3-knockdown cells with or without RAMP treatment (Figure 4C), suggesting that MA was not activated in the cells when the IFITM3 gene was knocked down. However, IFITM3 knockdown led to a cascade of effects causing increased membrane permeability that eventually led to cell death through activation of lysosomes and the CMA pathway.

\section{Activation of the CMA Pathway when Knockdown of IFITM3}

Cell viability was significantly inhibited and eventually caused cell death after IFITM3 knockdown. However, the MA pathway was not the main pathway responsible for this regulatory effect, and lysosomes were significantly activated, while the expression of key CMA pathway-related proteins was obviously increased. Based on these data, we examined apoptotic cells by staining the cells with calceinAM. The results showed no obvious apoptosis in IFITM3-knockdown cells (Supplementary Figure 3A-C), and no apoptotic bodies were detected by Hoechst 33342 staining (Supplementary Figure 3D-F).

Additionally, the results of western blot analysis showed significantly increased expression of the antiapoptotic protein BCL2 and decreased expression of P53 (Figure 5A) in IFITM3-knockdown cells, which suggested that the apoptotic pathway was not activated when IFITM3 was knocked down. Cell activity was greatly decreased, and the expression of CCND1, the main function of which is to promote cell proliferation, was significantly decreased in the knockdown group. Further study showed obviously decreased expression of ERK1/2 among the groups (Figure 5B), suggesting that the downregulation of IFITM3 resulted in decreased cell survival and that the ability of the cells to proliferate was severely reduced. Our further investigation focused on the MA and CMA pathways. Expression of the related proteins P62 and LAMP1 was significantly upregulated (Figure $5 A$ ), but there was no significant change in the MA pathway-related proteins ATG13, BECLIN1, ATG5, ATG7 and LC3A/B (Figure 5A). Meanwhile, the expression of LAMP2A and HSC70 (Figure 5 C) was significantly upregulated, which indicated and confirmed the activation of CMA in the cells after IFITM3 knockdown. There was also no significant difference in the expression of LC3A/B in IFITM3-knockdown cells (Figure 5D).

To study the generation of autophagic flux upon IFITM3 knockdown, stable LC3-GFP-mCherry-expressing mNRPCs were constructed. The results showed that no autophagic flux was generated in the cells (Figure 5D and Supplementary Figure 4). When IFITM3 was knocked down (Figure 6A-C), the results of 
immunohistochemical (IHC) analysis showed lightly increased expression of BCL2 (Figure 6D-F) and no significant changes in ATG7 expression (Figure 6G-I), while the expression of LAMP1 (Figure 6J-L), P62 (Figure 6M-0) and LAMP2A (Figure 6P-R) was significantly changed. The data were consistent with the WB results, indicating no activation of MA or the apoptotic pathway, while the CMA pathway was activated in IFITM3-knockdown cells. According to the above data, we believe that IFITM3 regulates cell viability mainly by regulating the CMA pathway, suggesting that the IFITM3 gene has a protective function that increases progenitor cell survival and self-renewal.

\section{Discussion}

Stem cell research and related cell therapies have developed rapidly around the world. In the field of cellular ophthalmology therapy, safety data have emerged from several phase I/II clinical trials of cell transplantation with retinal pigment epithelial (RPE) cells or MSCs [25-35]; however, some issues, such as host-graft rejection, validation over too short a duration (less than 12 months) and a lack of clinical significance, remain. Although RPC transplantation is considered a very promising approach[2, 36-38], its application is limited because of insufficient donor cells. Therefore, the need to obtain enough seed cells has become urgent. Previously, we established mNRPCs that can be cultured long-term in vitro and maintain their self-renewal property based on the combination of bFGF and CHIR99021[3]. In a further biological study, RNA-seq data showed the high expression of some genes that are expressed at low levels in adult retinal tissue, such as IFITM3, an intrinsic host factor with antiviral function[39, 40], immune function[41-44] and germ cell specification[45] that is localized in several cellular components, including the apical part of the cell, cell surface and endolysosomal membrane. Currently, limited reports on the role of IFITM3 in stem cells are available, and its specific function in the field is still unclear. We studied the function of the IFITM3 gene in mNRPCs by knocking it down. The mNRPCs showed significantly decreased cell viability and proliferation after IFITM3 knockdown. By examining the expression of CCND1, a key molecule in the regulation of cell cycle progression from $\mathrm{G} 1$ to $\mathrm{S}$ phase, we found that CCND1 was significantly decreased, suggesting that IFITM3 affects the proliferation of mNRPCs by regulating cell division. In addition, the expression of ERK $1 / 2$ was decreased accordingly after IFITM3 knockdown, indicating that IFITM3 regulates cell proliferation and may be associated with the MAPK pathway.

Subsequently, we performed GO and KEGG analyses of the RNA-seq results and found that IFITM3 is involved in regulating $\mathrm{MNRPC}$ proliferation by changing the structure and function of the cell membrane. Additionally, lysosomes showed a marked increase in abundance in this abnormal membrane system. Moreover, we found that knockdown of IFITM3 caused lysosome agglomeration in the cells. Knowing that lysosomes are a key factor in autophagy and important for regulating mTORC1 signaling[46-49], the main regulator of autophagy, especially MA, we studied changes in lysosomes and the autophagy pathway after RAMP treatment when IFITM3 was knocked down. The autophagy pathway is classified into three types: MA, $\mathrm{mA}[50-52]$ and $\mathrm{CMA}[52-55]$; these three pathways degrade damaged cells or organelles. We found that knockdown of IFITM3 inhibited cell proliferation and cell viability and significantly enhanced lysosome agglomeration, which was accompanied by an obvious increase in the 
key CMA-related molecules LAMP2A and HSC70. CMA is a lysosomal-dependent protein degradation pathway, consistent with this finding. We believe that IFITM3 regulates cell proliferation mainly through activation of the CMA pathway and that this regulation involves the activation and recruitment of lysosomes. Our results are consistent with the results of Cuervo's laboratory[55], which reported that CMA is required for the maintenance and function of hematopoietic stem cells (HSCs) as it contributes to protein quality control in quiescent steady-state HSCs. Our study suggests that CMA is very important for neural progenitor cells to maintain the homeostasis at basal activity, and the overactivation of CMA will cause cell death. Recent research results have shown that autophagy is closely related to many neurodegenerative diseases. In the future, further study of the relationship between IFITM3 and neuroretinal degeneration may provide new therapeutic insight into neuroretinal degenerative diseases.

\section{Conclusion}

In this report, we found that IFITM3 plays an important role in RPC proliferation by activating the CMA pathway, suggesting that the low-level activation of CMA is important in maintaining the homeostasis of RPCs, while the overactivation of CMA leads to cell death.

\section{Abbreviations}

BC: blank control

CCND1: Cyclin D1

CMA chaperone-mediated autophagy

DEG: differentially expressed gene

ECM: extracellular matrix

ER: endoplasmic reticulum

GO: Gene Ontology

IFITM3: interferon-induced transmembrane protein 3

KEGG: Kyoto Encyclopedia of Genes and Genomes

LAMP1: lysosome-associated membrane protein 1

mA: microautophagy

mNRPC: mouse neural retinal progenitor cell

MSC: mesenchymal stem cells 
OD: optical density

PFA: paraformaldehyde

RAMP: rapamycin

RPC: retinal progenitor cell

SC: scramble control

UMAP: uniform manifold approximation and projection

\section{Declarations}

\section{Consent for publication}

Not applicable.

\section{Ethics approval}

All animal procedures were performed according to the institutional guidelines and the Guide for the Care and Use of Laboratory Animals issued by the $\mathrm{NIH}$ and the guidelines of the animal experimentation ethics committee of Tongji University, and in accordance with the Association for Research in Vision and Ophthalmology Statement for the use of Animals in Ophthalmic and Vision Research.

\section{Availability of Data and Material}

RNA-seq data generated in the study can be accessed at the Gene Expression Omnibus under accession code GSE176472(https://www.ncbi.nlm.nih.gov/geo/query/acc.cgi?acc=GSE176472). All other data are included within the article and its additional files.

\section{Authors' Contributions}

$\mathrm{CJ}$ and LL conceived and designed the experiments, performed the experiments, analyzed the data, and wrote the paper. QO and ZW collected and analyzed the RNA-Seq data. CJ, QO, HT and G-T X contributed to design the experiment, financial support, manuscript writing and final approval of manuscript. TW, JC and TW performed the experiments. JZ, YW, J-Y X, FG, JW and JL contributed to reagents/materials/analysis tools. All authors have read and approved the manuscript.

\section{Conflict of interest}

The authors declare that they have no competing interests.

\section{Funding}


This paper was supported by the National Natural Science Foundation of China $(32070719,81770942)$, and China Postdoctoral Science Foundation (2019M661631).

\section{Acknowledgements}

Not applicable.

\section{References}

1. Wang Z, Gao F, Zhang M, Zheng Y, Zhang F, Xu L, Cao L, He W: Intravitreal Injection of Human Retinal Progenitor Cells for Treatment of Retinal Degeneration. Med Sci Monit 2020, 26:e921184.

2. Liu Y, Chen SJ, Li SY, Qu LH, Meng XH, Wang Y, Xu HW, Liang ZQ, Yin ZQ: Long-term safety of human retinal progenitor cell transplantation in retinitis pigmentosa patients. Stem Cell Res Ther 2017, 8(1):209.

3. Jin C, Ou Q, Li Z, Wang J, Zhang J, Tian H, Xu JY, Gao F, Lu L, Xu GT: The combination of bFGF and CHIR99021 maintains stable self-renewal of mouse adult retinal progenitor cells. Stem Cell Res Ther 2018, 9(1):346.

4. Ohsumi Y: Molecular mechanism of autophagy in yeast, Saccharomyces cerevisiae. Philos Trans $R$ Soc Lond B Biol Sci 1999, 354(1389):1577-1580; discussion 1580-1571.

5. Goyal A, Neill T, Owens RT, Schaefer L, lozzo RV: Decorin activates AMPK, an energy sensor kinase, to induce autophagy in endothelial cells. Matrix Bio/ 2014, 34:46-54.

6. Ashoor R, Yafawi R, Jessen B, Lu S: The contribution of lysosomotropism to autophagy perturbation. PLoS One 2013, 8(11):e82481.

7. Brenner C, Galluzzi L, Kepp O, Kroemer G: Decoding cell death signals in liver inflammation. $J$ Hepatol 2013, 59(3):583-594.

8. Kim MJ, Febbraro D, Farkona S, Gillmore T, Son JE, Regeenes R, Chang HH, Pollock-Tahiri E, Yang J, Park YJ et al: Distinct roles of UVRAG and EGFR signaling in skeletal muscle homeostasis. $\mathrm{Mol}$ Metab 2021, 47:101185.

9. Tseng WC, Lee PY, Tsai MT, Chang FP, Chen NJ, Chien CT, Hung SC, Tarng DC: Hypoxic mesenchymal stem cells ameliorate acute kidney ischemia-reperfusion injury via enhancing renal tubular autophagy. Stem Cell Res Ther 2021, 12(1):367.

10. Tang J, Ye Z, Liu Y, Zhou M, Huang L, Mo Q, Su X, Qin C: Autophagy-deficiency in bone marrow mononuclear cells from patients with myasthenia gravis: a possible mechanism of pathogenesis. Int J Neurosci 2021, 131(3):239-253.

11. Savio-Silva C, Soinski-Sousa PE, Simplicio-Filho A, Bastos RMC, Beyerstedt S, Rangel EB: Therapeutic Potential of Mesenchymal Stem Cells in a Pre-Clinical Model of Diabetic Kidney Disease and Obesity. Int J Mol Sci 2021, 22(4).

12. Brass AL, Huang IC, Benita Y, John SP, Krishnan MN, Feeley EM, Ryan BJ, Weyer JL, van der Weyden L, Fikrig E et al: The IFITM proteins mediate cellular resistance to influenza A H1N1 virus, West Nile 
virus, and dengue virus. Cell 2009, 139(7):1243-1254.

13. Schoggins JW, Wilson SJ, Panis M, Murphy MY, Jones CT, Bieniasz P, Rice CM: A diverse range of gene products are effectors of the type I interferon antiviral response. Nature 2011, 472(7344):481485.

14. Weidner JM, Jiang D, Pan XB, Chang J, Block TM, Guo JT: Interferon-induced cell membrane proteins, IFITM3 and tetherin, inhibit vesicular stomatitis virus infection via distinct mechanisms. $J$ Virol 2010, 84(24):12646-12657.

15. Yount JS, Moltedo B, Yang YY, Charron G, Moran TM, Lopez CB, Hang HC: Palmitoylome profiling reveals S-palmitoylation-dependent antiviral activity of IFITM3. Nat Chem Biol 2010, 6(8):610-614.

16. Galvin HD, Husain M: Influenza A virus-induced host caspase and viral PA-X antagonize the antiviral host factor, histone deacetylase 4. J Biol Chem 2019, 294(52):20207-20221.

17. Sun Q, Lei N, Lu J, Gao RB, Li Z, Liu LQ, Sun Y, Guo JF, Wang DY, Shu YL: Interferon-induced Transmembrane Protein 3 Prevents Acute Influenza Pathogenesis in Mice. Biomed Environ Sci 2020, 33(5):295-305.

18. Evans SS, Collea RP, Leasure JA, Lee DB: IFN-alpha induces homotypic adhesion and Leu-13 expression in human B lymphoid cells. J Immunol 1993, 150(3):736-747.

19. Smith RA, Young J, Weis JJ, Weis JH: Expression of the mouse fragilis gene products in immune cells and association with receptor signaling complexes. Genes Immun 2006, 7(2):113-121.

20. Saitou M, Payer B, Lange UC, Erhardt S, Barton SC, Surani MA: Specification of germ cell fate in mice. Philos Trans R Soc Lond B Biol Sci 2003, 358(1436):1363-1370.

21. Tanaka SS, Nagamatsu G, Tokitake Y, Kasa M, Tam PP, Matsui Y: Regulation of expression of mouse interferon-induced transmembrane protein like gene-3, Ifitm3 (mil-1, fragilis), in germ cells. Dev Dyn 2004, 230(4):651-659.

22. Tanaka SS, Yamaguchi YL, Tsoi B, Lickert H, Tam PP: IFITM/Mil/fragilis family proteins IFITM1 and IFITM3 play distinct roles in mouse primordial germ cell homing and repulsion. Dev Cell 2005, 9(6):745-756.

23. Clark BS, Stein-O'Brien GL, Shiau F, Cannon GH, Davis-Marcisak E, Sherman T, Santiago CP, Hoang TV, Rajaii F, James-Esposito RE et al: Single-Cell RNA-Seq Analysis of Retinal Development Identifies NFI Factors as Regulating Mitotic Exit and Late-Born Cell Specification. Neuron 2019, 102(6):11111126 e1115.

24. Butler A, Hoffman P, Smibert P, Papalexi E, Satija R: Integrating single-cell transcriptomic data across different conditions, technologies, and species. Nat Biotechnol 2018, 36(5):411-420.

25. Algvere PV, Berglin L, Gouras P, Sheng Y, Kopp ED: Transplantation of RPE in age-related macular degeneration: observations in disciform lesions and dry RPE atrophy. Graefes Arch Clin Exp Ophthalmol 1997, 235(3):149-158.

26. da Cruz L, Fynes K, Georgiadis O, Kerby J, Luo YH, Ahmado A, Vernon A, Daniels JT, Nommiste B, Hasan SM et al: Phase 1 clinical study of an embryonic stem cell-derived retinal pigment epithelium patch in age-related macular degeneration. Nat Biotechnol 2018, 36(4):328-337. 
27. Humayun MS, de Juan E, Jr., del Cerro M, Dagnelie G, Radner W, Sadda SR, del Cerro C: Human neural retinal transplantation. Invest Ophthalmol Vis Sci 2000, 41(10):3100-3106.

28. Radtke ND, Aramant RB, Petry HM, Green PT, Pidwell DJ, Seiler MJ: Vision improvement in retinal degeneration patients by implantation of retina together with retinal pigment epithelium. $\mathrm{Am} \mathrm{J}$ Ophthalmol 2008, 146(2):172-182.

29. Schwartz SD, Hubschman JP, Heilwell G, Franco-Cardenas V, Pan CK, Ostrick RM, Mickunas E, Gay R, Klimanskaya I, Lanza R: Embryonic stem cell trials for macular degeneration: a preliminary report. Lancet 2012, 379(9817):713-720.

30. Schwartz SD, Regillo CD, Lam BL, Eliott D, Rosenfeld PJ, Gregori NZ, Hubschman JP, Davis JL, Heilwell G, Spirn M et al: Human embryonic stem cell-derived retinal pigment epithelium in patients with age-related macular degeneration and Stargardt's macular dystrophy: follow-up of two openlabel phase 1/2 studies. Lancet 2015, 385(9967):509-516.

31. Song WK, Park KM, Kim HJ, Lee JH, Choi J, Chong SY, Shim SH, Del Priore LV, Lanza R: Treatment of macular degeneration using embryonic stem cell-derived retinal pigment epithelium: preliminary results in Asian patients. Stem Cell Reports 2015, 4(5):860-872.

32. Siqueira RC, Messias A, Voltarelli JC, Scott IU, Jorge R: Intravitreal injection of autologous bone marrow-derived mononuclear cells for hereditary retinal dystrophy: a phase I trial. Retina 2011, 31(6):1207-1214.

33. Siqueira RC, Messias A, Voltarelli JC, Messias K, Arcieri RS, Jorge R: Resolution of macular oedema associated with retinitis pigmentosa after intravitreal use of autologous BM-derived hematopoietic stem cell transplantation. Bone Marrow Transplant 2013, 48(4):612-613.

34. Siqueira RC, Messias A, Messias K, Arcieri RS, Ruiz MA, Souza NF, Martins LC, Jorge R: Quality of life in patients with retinitis pigmentosa submitted to intravitreal use of bone marrow-derived stem cells (Reticell -clinical trial). Stem Cell Res Ther 2015, 6:29.

35. Satarian L, Nourinia R, Safi S, Kanavi MR, Jarughi N, Daftarian N, Arab L, Aghdami N, Ahmadieh H, Baharvand H: Intravitreal Injection of Bone Marrow Mesenchymal Stem Cells in Patients with Advanced Retinitis Pigmentosa; a Safety Study. J Ophthalmic Vis Res 2017, 12(1):58-64.

36. Hendrickson A, Bumsted-O'Brien K, Natoli R, Ramamurthy V, Possin D, Provis J: Rod photoreceptor differentiation in fetal and infant human retina. Exp Eye Res 2008, 87(5):415-426.

37. Gu P, Harwood LJ, Zhang X, Wylie M, Curry WJ, Cogliati T: Isolation of retinal progenitor and stem cells from the porcine eye. Mol Vis 2007, 13:1045-1057.

38. Tang Z, Jiang F, Zhang Y, Zhang Y, YuanYang, Huang X, Wang Y, Zhang D, Ni N, Liu F et al: Musselinspired injectable hydrogel and its counterpart for actuating proliferation and neuronal differentiation of retinal progenitor cells. Biomaterials 2019, 194:57-72.

39. Li C, Du S, Tian M, Wang Y, Bai J, Tan P, Liu W, Yin R, Wang M, Jiang Y et al: The Host Restriction Factor Interferon-Inducible Transmembrane Protein 3 Inhibits Vaccinia Virus Infection. Front Immunol 2018, 9:228. 
40. Smith SE, Busse DC, Binter S, Weston S, Diaz Soria C, Laksono BM, Clare S, Van Nieuwkoop S, Van den Hoogen BG, Clement M et al: Interferon-Induced Transmembrane Protein 1 Restricts Replication of Viruses That Enter Cells via the Plasma Membrane. J Virol 2019, 93(6).

41. Wee YS, Roundy KM, Weis JJ, Weis JH: Interferon-inducible transmembrane proteins of the innate immune response act as membrane organizers by influencing clathrin and v-ATPase localization and function. Innate Immun 2012, 18(6):834-845.

42. Verrier ER, Colpitts CC, Bach C, Heydmann L, Zona L, Xiao F, Thumann C, Crouchet E, Gaudin R, Sureau C et al: Solute Carrier NTCP Regulates Innate Antiviral Immune Responses Targeting Hepatitis C Virus Infection of Hepatocytes. Cell Rep 2016, 17(5):1357-1368.

43. Campbell RA, Schwertz H, Hottz ED, Rowley JW, Manne BK, Washington AV, Hunter-Mellado R, Tolley ND, Christensen $M$, Eustes AS et al: Human megakaryocytes possess intrinsic antiviral immunity through regulated induction of IFITM3. Blood 2019, 133(19):2013-2026.

44. Nutsford AN, Galvin HD, Ahmed F, Husain M: The Class IV human deacetylase, HDAC11, exhibits antiinfluenza A virus properties via its involvement in host innate antiviral response. Cell Microbiol 2019, 21(4):e12989.

45. Young JC, Dias VL, Loveland KL: Defining the window of germline genesis in vitro from murine embryonic stem cells. Biol Reprod 2010, 82(2):390-401.

46. Atwood DJ, Pokhrel D, Brown CN, Holditch SJ, Bachu DM, Thorburn A, Hopp K, Edelstein CL: Increased mTOR and suppressed autophagic flux in the heart of a hypomorphic Pkd1 mouse model of autosomal dominant polycystic kidney disease. Cell Signa/2020, 74:109730.

47. Sironi J, Aranda E, Nordstrom LU, Schwartz EL: Lysosome Membrane Permeabilization and Disruption of the Molecular Target of Rapamycin (mTOR)-Lysosome Interaction Are Associated with the Inhibition of Lung Cancer Cell Proliferation by a Chloroquinoline Analog. Mol Pharmacol 2019, 95(1):127-138.

48. Chen Y, Xu S, Wang N, Ma Q, Peng P, Yu Y, Zhang L, Ying Z, Wang H: Dynasore Suppresses mTORC1 Activity and Induces Autophagy to Regulate the Clearance of Protein Aggregates in Neurodegenerative Diseases. Neurotox Res 2019, 36(1):108-116.

49. Hong Z, Pedersen NM, Wang L, Torgersen ML, Stenmark H, Raiborg C: Ptdlns3P controls mTORC1 signaling through lysosomal positioning. J Cell Biol 2017, 216(12):4217-4233.

50. Li M, Guo J, Wang H, Li Y: Involvement of Mitochondrial Dynamics and Mitophagy in SevofluraneInduced Cell Toxicity. Oxid Med Cell Longev 2021, 2021:6685468.

51. Lizama BN, Chu CT: Neuronal autophagy and mitophagy in Parkinson's disease. Mol Aspects Med 2021:100972.

52. Zheng J, Wei S, Xiao T, Li G: LC3B/p62-mediated mitophagy protects A549 cells from resveratrolinduced apoptosis. Life Sci 2021, 271:119139.

53. Tokarchuk I, Janser FA, Schlafli AM, Pinto MT, Humbert M, Niklaus NJ, Berezowska S, Langer R, Tschan MP: Increased LAMP2A levels correlate with a shorter disease-free survival of HER2 negative 
breast cancer patients and increased breast cancer cell viability. Biochem Biophys Res Commun 2021, 569:47-53.

54. Xu X, Sun Y, Cen X, Shan B, Zhao Q, Xie T, Wang Z, Hou T, Xue Y, Zhang M et al: Metformin activates chaperone-mediated autophagy and improves disease pathologies in an Alzheimer disease mouse model. Protein Cell 2021.

55. Dong S, Wang Q, Kao YR, Diaz A, Tasset I, Kaushik S, Thiruthuvanathan V, Zintiridou A, Nieves E, Dzieciatkowska $\mathrm{M}$ et al: Chaperone-mediated autophagy sustains haematopoietic stem-cell function. Nature 2021, 591(7848):117-123.

\section{Figures}



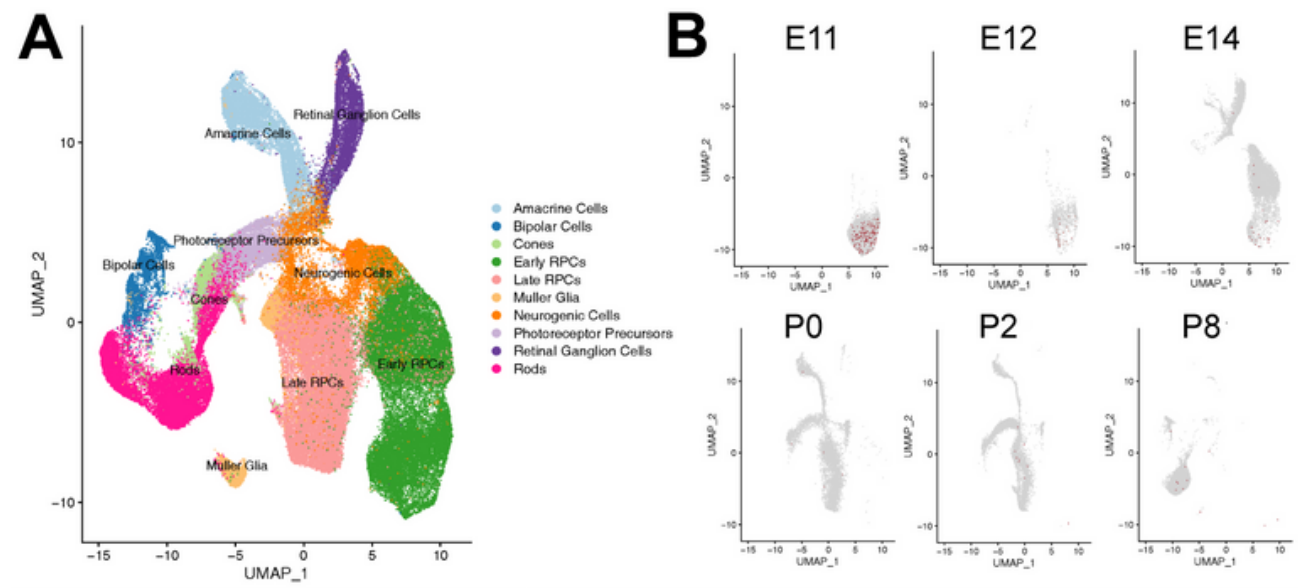

E16
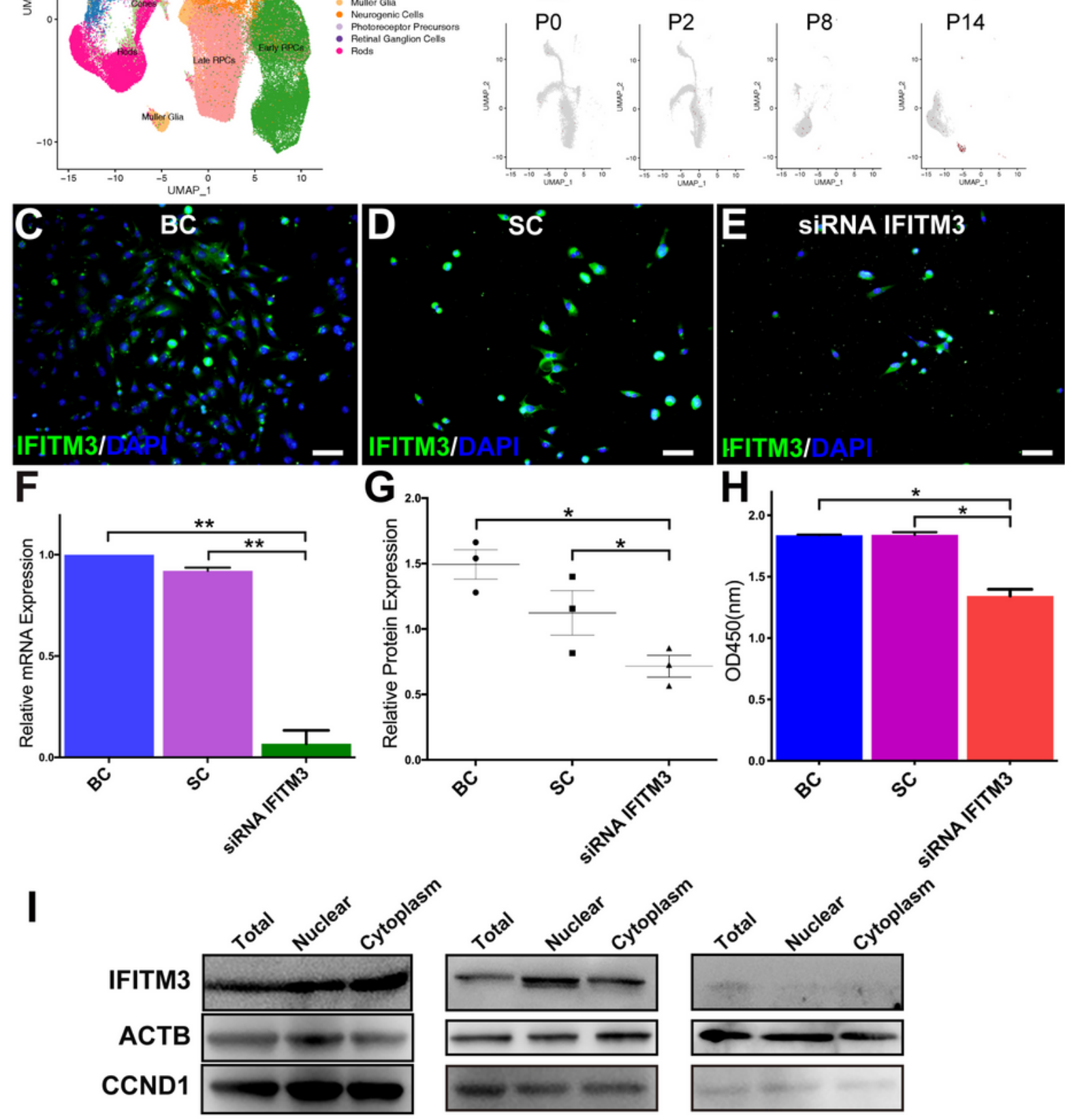

BC

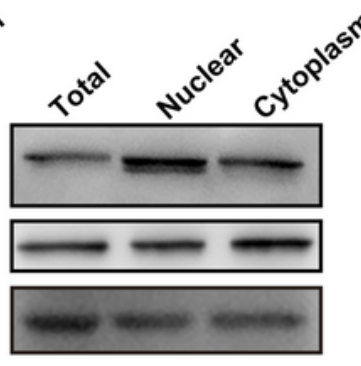

SC

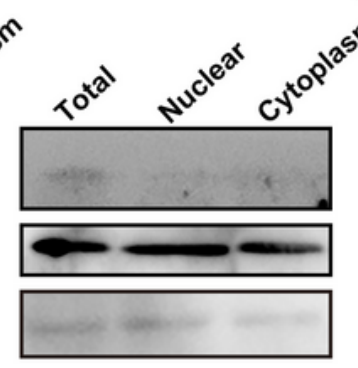

SIRNA IFITM3

\section{Figure 1}

mNRPC proliferation was greatly inhibited after IFITM3 knockdown for 48h. (A) DimPlot showed the cell types in the murine retina during embryonic stage and postnatal stage. (B) FeaturePlot showed the expression and distribution of IFITM3 at E11, E12, E14, E16, P0, P2, P8 and P14. (C-E) Immunofluorescence assays showed decreased expression of IFITM3 in mNRPCs. Magnification: $\times 200$; scale bar: $100 \mu \mathrm{m}$. (F) qRT-PCR showed decreased expression of IFITM3 in mNRPCs. (G) The results of 
WB assays were consistent with the qPCR results. $(\mathrm{H})$ The CCK-8 assay was used to assess cells after IFITM3 knockdown for $48 \mathrm{~h}$. Data are presented as the mean $\pm \mathrm{SD}(\mathrm{n}=3)$. *: $\mathrm{P}<0.05$; $*$ : $\mathrm{P}<0.01$ (oneway ANOVA and Sidak's multiple comparisons test). (I) Cell division-related protein expression was decreased in IFITM3-knockdown cells, as determined by WB assays. The results are representative of at least three independent experiments, and representative blots are shown. Abbreviation: BC, blank control; SC, scramble control.

\section{A}

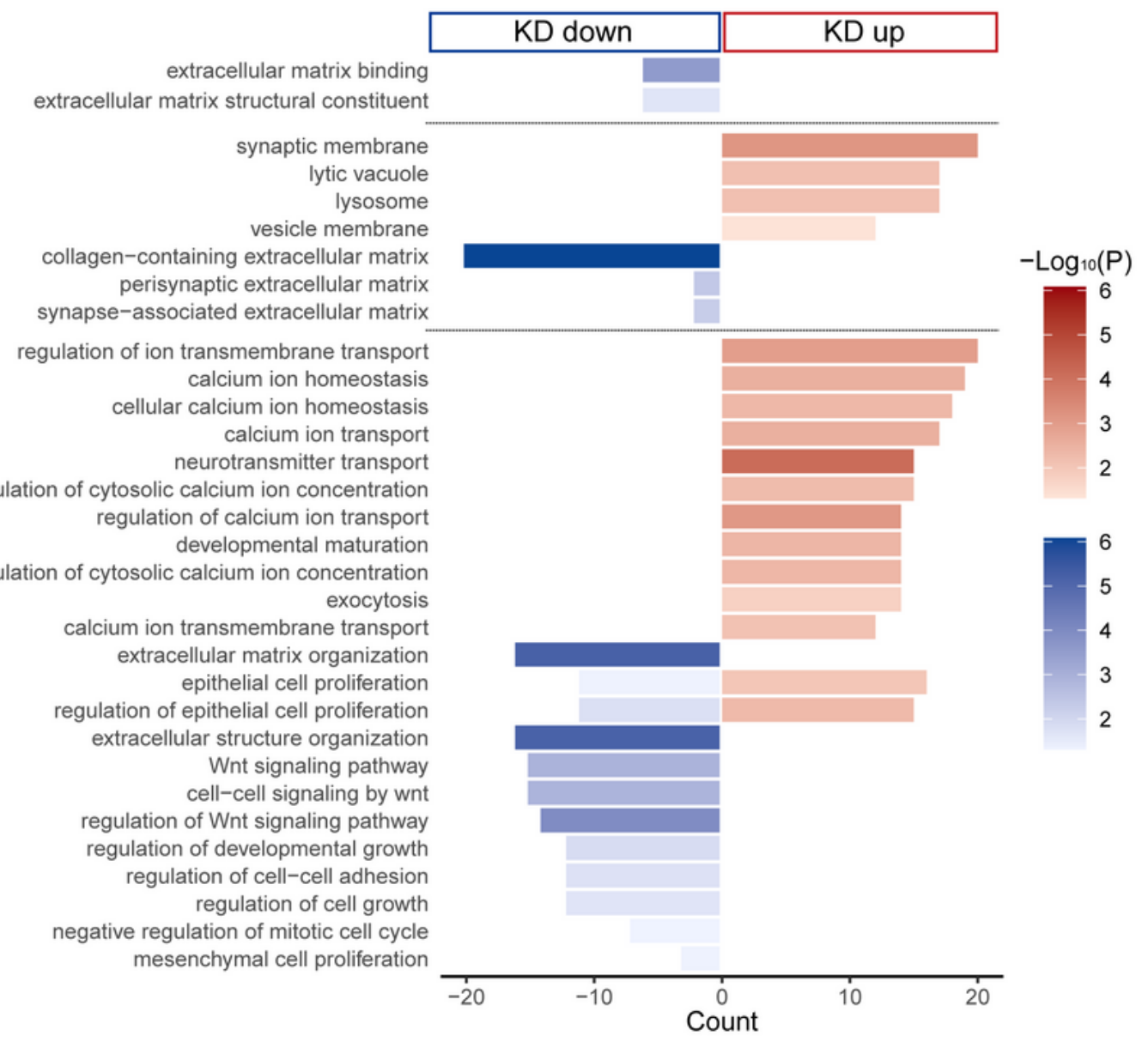

\section{B}
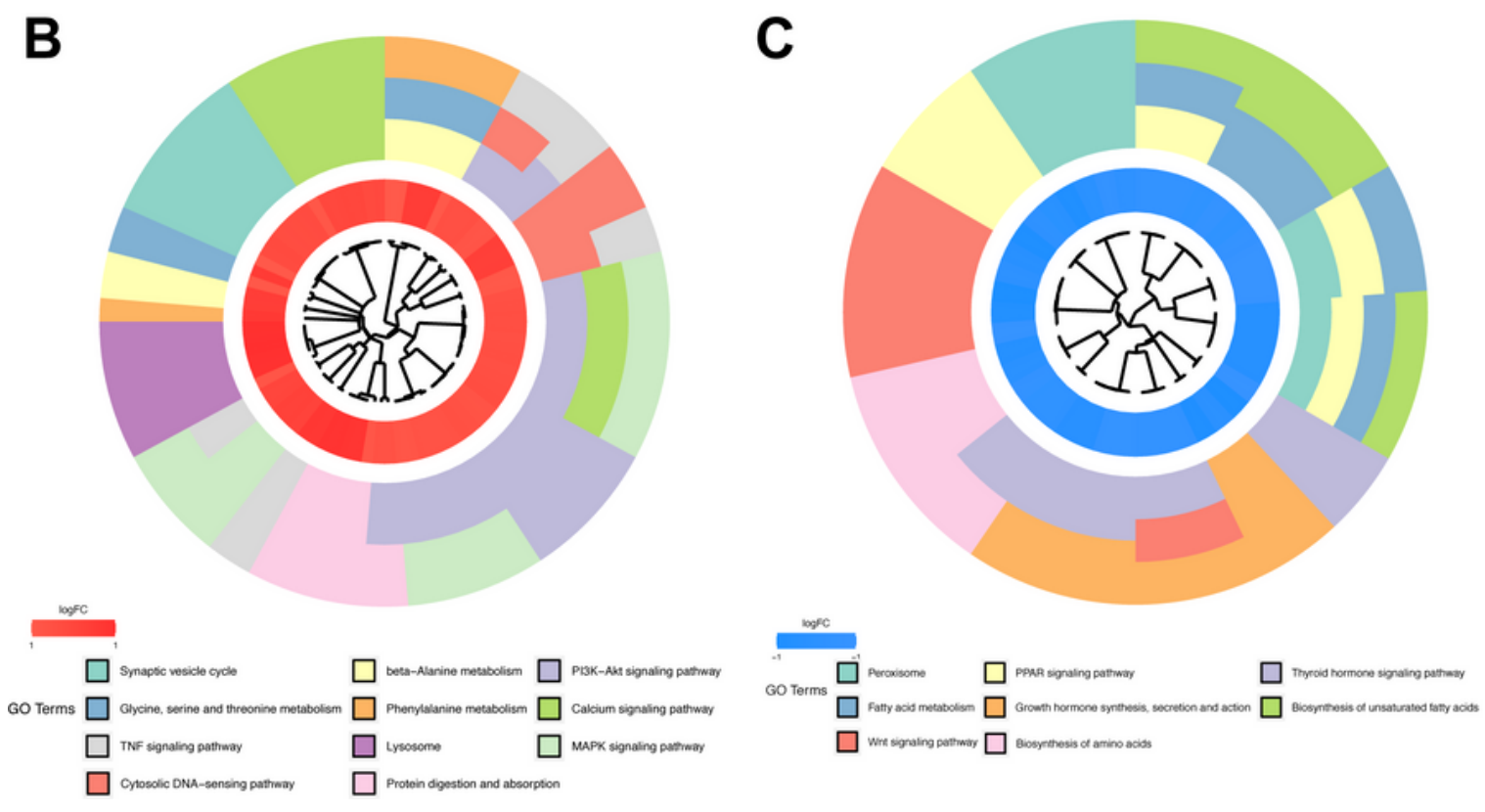

Figure 2 
High-throughput sequencing analysis of IFITM3-knockdown cells. (A) GO analysis revealed the expression of genes in IFITM3-knockdown cells. (B-C) KEGG Chord analysis showed the signaling pathways enriched in differentially expressed genes (DEGs).
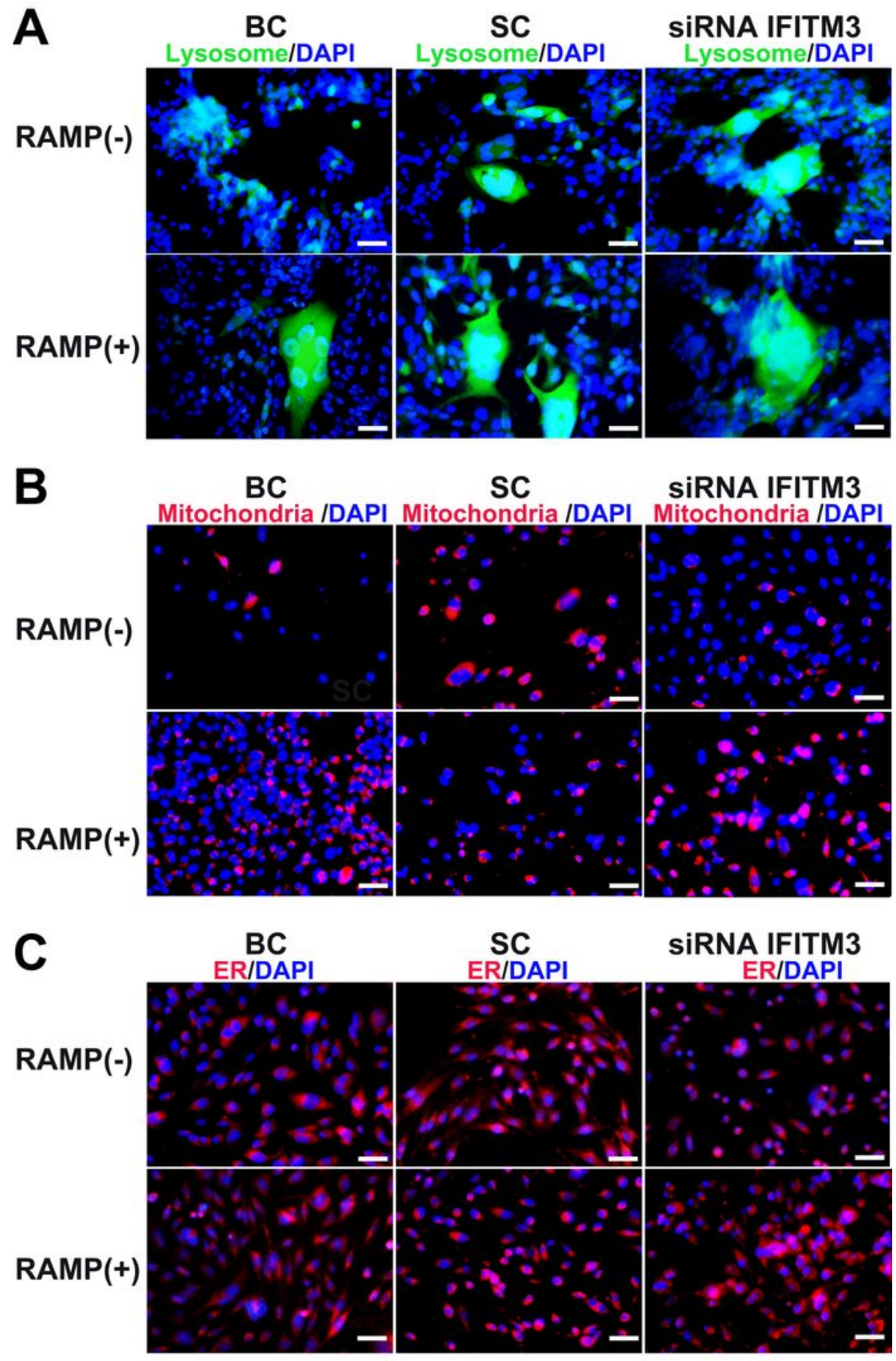

\section{Figure 3}

Organelles involved in the effects of IFITM3 knockdown in cells with or without rapamycin treatment. (A) Fluorescent images showing the localization of lysosomes in cells treated with $50 \mathrm{nM}$ LysoTracker for 30 
min. (B) Fluorescence images showing the localization of mitochondria in cells treated with $50 \mathrm{nM}$ MitoTracker for $30 \mathrm{~min}$. (C) Fluorescence images showing localization of after the ER in cells treated with $1 \mu \mathrm{M}$ ER-Tracker for 30 min. Magnification: × 400; scale bar: $50 \mu \mathrm{m}$. Abbreviation: BC, blank control; SC, scramble control.

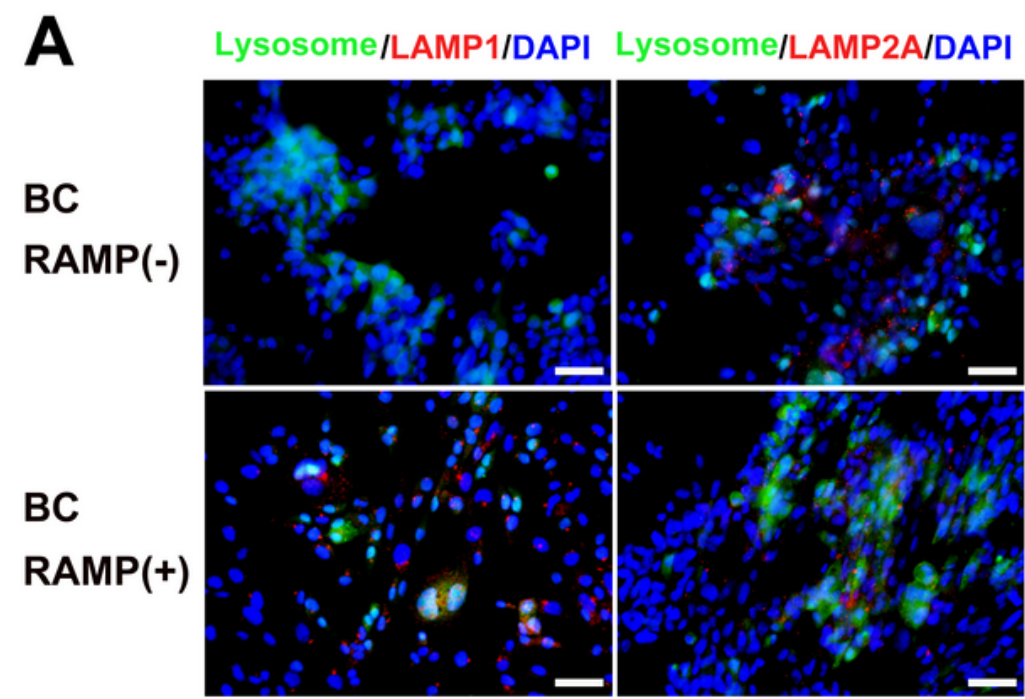

B

SC

RAMP(-)

SC

RAMP(+)

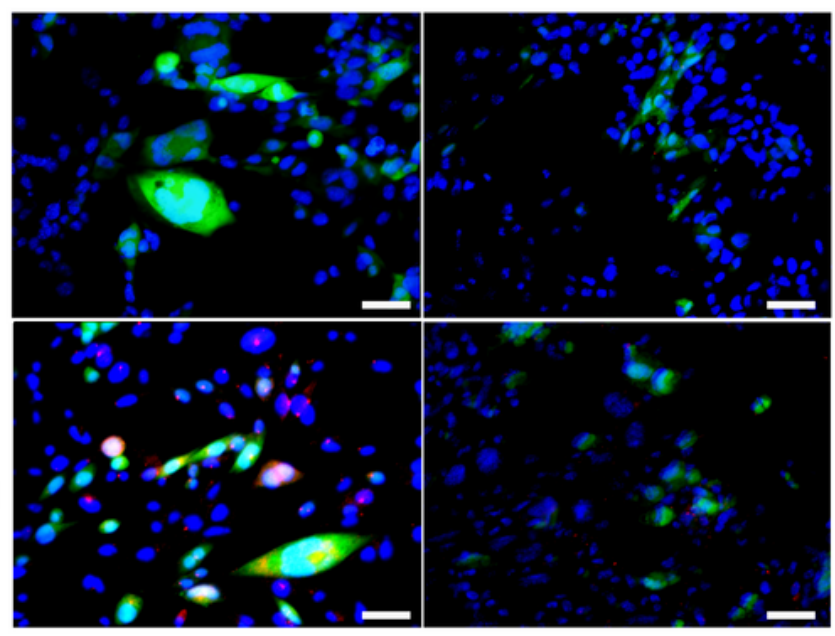

C

SiRNA

IFITM3

RAMP(-)

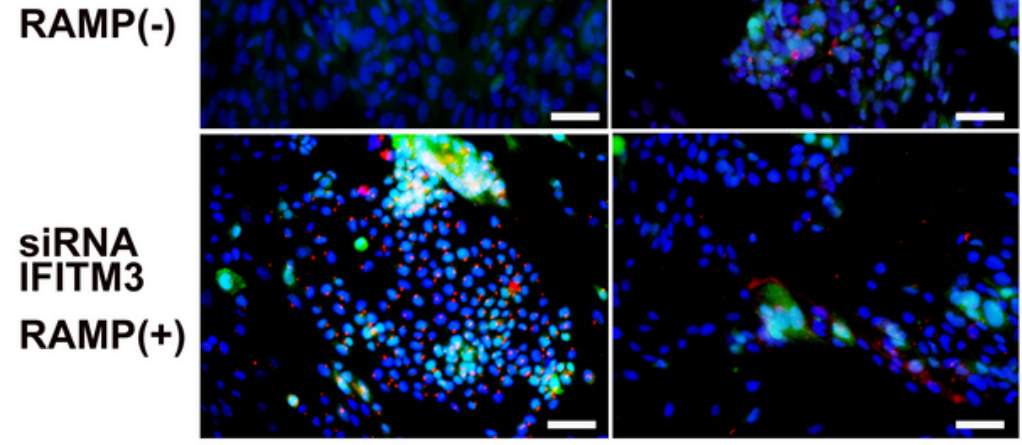

Figure 4 
Lysosome activation in IFITM3-knockdown cells treated with or without rapamycin. Immunofluorescence staining of LAMP1 and LAMP2A in cells treated with or without RAMP for 48 hours that were labeled with $50 \mathrm{nM}$ LysoTracker for $30 \mathrm{~min}$. (A) The blank control group, (B) Scramble control group and (C) IFITM3knockdown group. Abbreviation: BC, blank control; SC, scramble control.

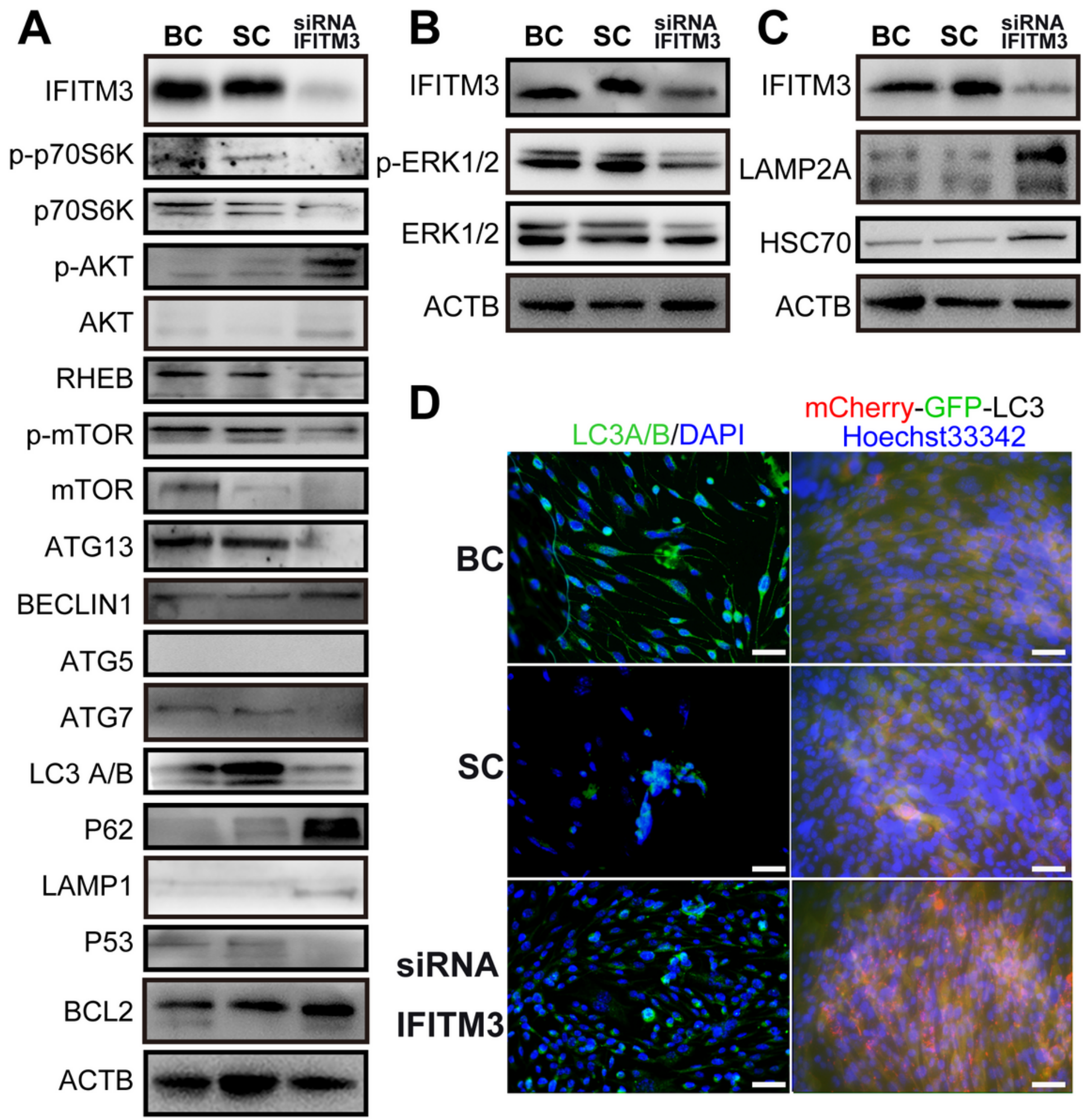

Figure 5 
IFITM3 increases the survival and maintains the proliferation of mNRPCs mainly through the CMA signaling pathway. (A) The expression of key pathway-related proteins in cells after IFITM3 knockdown, including MA. (B) The expression of ERK1/2 was significantly decreased in IFITM3-knockdown cells. (C) CMA was activated, accompanied by increased expression of LAMP2A and HSC70 in cells in which the IFITM3 gene was knocked down. The results are representative of at least three independent experiments, and representative blots are shown. (D) Results of IHC analysis of LC3A/B and fluorescent images showing mCherry-GFP-LC3 expression after IFITM3 knockdown used to assay autophagic flux in the cells. Magnification: $\times 400$; scale bar: $50 \mu \mathrm{m}$. 


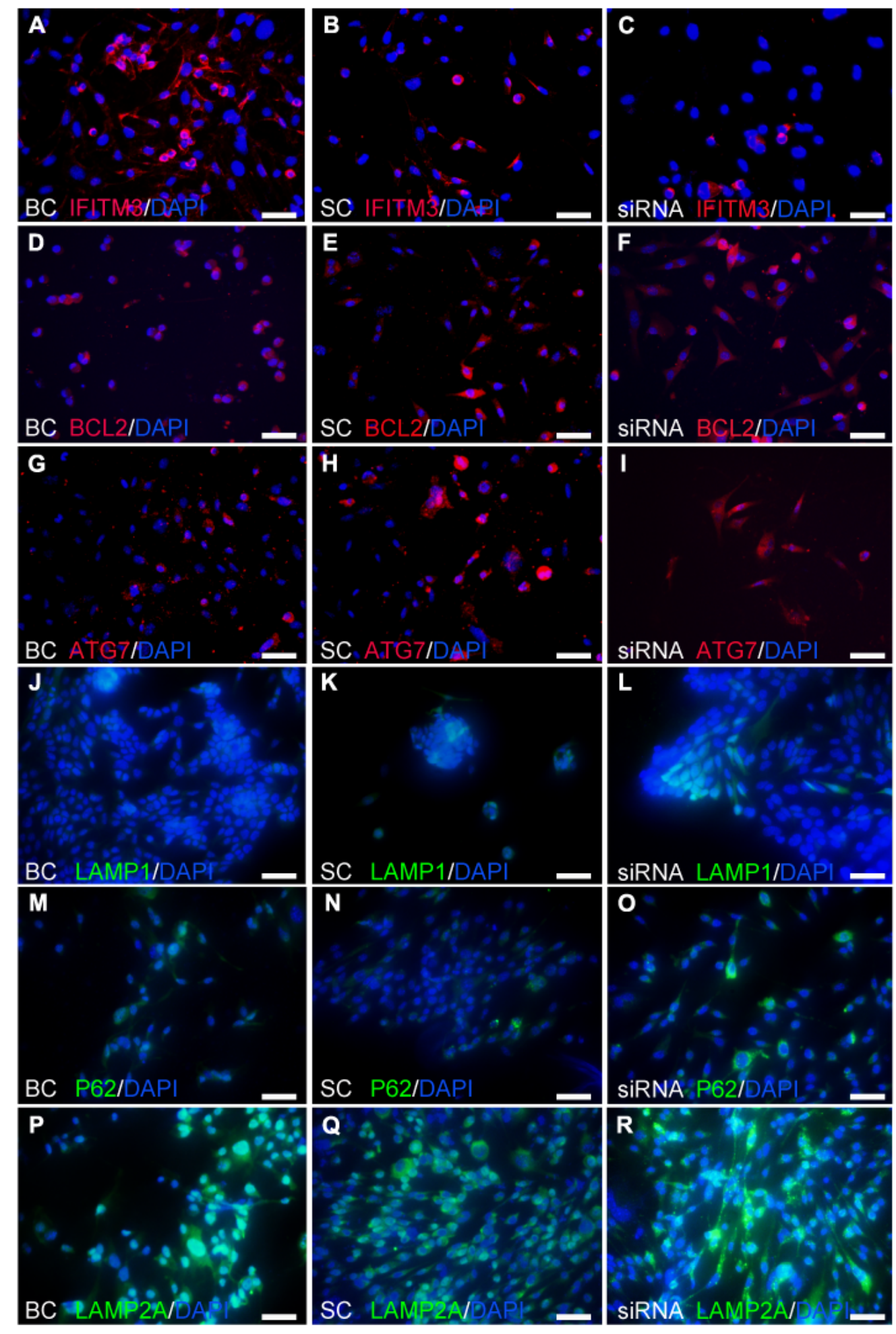

\section{Figure 6}

IHC assay upon IFITM3 knockdown. (A-C) Immunofluorescence staining of IFITM3 in the different groups. (D-F) Expression of the classic apoptosis pathway-related protein BCL2. (G-O) MA pathway related proteins: ATG7, LAMP1 and P62. (P-R) The expression of the CMA pathway protein LAMP2A was consistent with the WB results. The results are representative of at least three independent experiments 
and representative views are shown. Magnification: × 400; scale bar: $50 \mu \mathrm{m}$. Abbreviation: BC, blank control; SC, scramble control.

\section{Supplementary Files}

This is a list of supplementary files associated with this preprint. Click to download.

- InstructionofOriginalGelFiles.docx

- SupplementaryFigure1.tif

- SupplementaryFigure2.tif

- SupplementaryFigure3.tif

- SupplementaryFigure4.tif

- SupplementaryMaterialSCRT.docx 